\title{
Integración de Tecnologías Energéticamente Eficientes en Sistemas de Climatización Operados con Energía Térmica
}

\section{Integration of Energy Efficient Technologies in HVAC systems Operated with Thermal Energy}

\author{
J. A. Cardona-Gil ${ }^{1,2}$, J. H. Gallego Orrego ${ }^{1}$, C. A. Isaza Roldán ${ }^{1}$, \\ R. Torres Salazar ${ }^{1}$ y D. A. López Chejne ${ }^{1}$
}

Recibido: 02 de febrero 2017

Aceptado: 13 de mayo 2017

\section{Resumen}

Con el artículo se pretende mostrar el desarrollo de un proyecto demostrativo y educativo de un sistema de climatización operado con energía solar, en el cual se usan tecnologías complementarias energéticamente eficientes que potencien su desempeño dentro del bloque de registro de la Universidad Pontificia Bolivariana (UPB), así mismo potenciar la investigación, desarrollo e inversión para conseguir una alta penetración de tecnologías de climatización eficiente y limpia. El desarrollo se ha dividido en 4 grandes fases, que inician con la simulación del sistema de refrigeración, en el cual se ha utilizado el software TRNSYS ${ }^{\circ}$; dimensionamiento y diseño básico del sistema de refrigeración; búsqueda y selección de equipos p.e. bombas; máquina de absorción, colectores solares, entre otros; diseño de detalle de un sistema de climatización con energía solar térmica, en el cual se hace uso de un equipo

1 Grupo de Energía y Termodinámica, Centro de Investigación, Desarrollo y Calidad en Refrigeración y Climatización, Facultad de Ingeniería Mecánica, Universidad Pontificia Bolivariana, P. O. 56006,

e-mail: cesar.isaza@upb.edu.co,web: http://www.upb.edu.co/

2 Jefatura de Laboratorios, Laboratorio de Ingeniería Eléctrica y Electrónica - Ensayos a la Industria, Facultad de Ingeniería Eléctrica y Electrónica, Universidad Pontificia Bolivariana, P. 0. 56006, e-mail: jorgeandres.cardona@upb.edu.co, web: http://www.upb.edu.co/ 
de enfriamiento de agua por absorción, paneles radiantes y enfriador evaporativo indirecto. Entre los resultados obtenidos se encuentra el dimensionamiento y diseño detallado del sistema y la adquisición del equipo de absorción y enfriamiento evaporativo soportados en un diseño simulado al emplear los softwares TRNSYS ${ }^{\circledR}$ y MATLAB ${ }^{\circledR}$. Lo anterior permitió determinar los niveles de carga adecuados para cada uno de los equipos involucrados en el sistema, además de asegurar los parámetros de calidad de potencia para atender los requerimientos de energía eléctrica y aire acondicionado.

Palabras clave: Eficiencia Energética, Refrigeración por Absorción, Refrigeración Solar Térmica.

\section{Abstract}

This paper intends to show the development of a demonstrative and educational project of a system of air conditioning operated with solar energy, in which are used complementary energy efficient technologies that enhance its performance within the block of registration of the UPB, Research, development and investment to achieve a high penetration of efficient and clean air conditioning technologies. The development has been divided into 4 large phases, which start with the simulation of the refrigeration system, in which the software TRNSYS ${ }^{\circledR}$ has been used; Sizing and basic design of the refrigeration system; Search and selection of equipment p.e. pumps; Absorption machine, solar collectors, among others; Detail design of an air conditioning system with solar thermal energy, in which use is made of water cooling equipment by absorption, radiant panels and indirect evaporative cooler. Among the results obtained are the sizing and detailed design of the system and the acquisition of the absorption and evaporative cooling equipment supported in a simulated design when using the software TRNSYS ${ }^{\circledR}$ and MATLAB ${ }^{\circledR}$. This allowed to determine the appropriate load levels for each of the equipment involved in the system, in addition to ensuring the parameters of power quality to meet the requirements of electric power and air conditioning.

Keywords: Energy Efficiency, Absorption Refrigeration, Solar Thermal Refrigeration.

\section{Introducción}

os sistemas de refrigeración por absorción de simple efecto (con un sólo generador), accionados con energía solar requieren de la unión de varios componentes para que se obtenga un adecuado funcionamiento. Entre los componentes principales se encuentran: los Colectores Solares, la Máquina de Absorción, la Torre de Enfriamiento, un Tanque de Agua Caliente, un Calentador Auxiliar, un Tanque de Agua Helada y el sistema de control.

El propósito de un sistema de control en una planta de refrigeración es proporcionar un funcionamiento automático para evitar el costo de mano de obra de los operadores o donde el control es demasiado complejo para 
el funcionamiento manual [ ${ }^{[1]}$. En varias partes del mundo se han desarrollado este tipo de sistemas de aire acondicionado operados con energía solar mediante un ciclo de refrigeración por absorción, es el caso de Puerto Rico, en donde Meza et al. ${ }^{[2]}$ describen una instalación experimental compuesta de un Chiller de Absorción 35 kW (10 TR), movido por un arreglo de 113 m² de colector de placa plana de superficie selectiva, un estanque de almacenamiento de $5.7 \mathrm{~m}^{3}$, una torre de refrigeración de $84 \mathrm{~kW}$ de capacidad y con la aplicación de un control ON-OFF para la estabilidad y manipulación del sistema.

Los sistemas de refrigeración por absorción, en su mayoría, son controlados por controladores de encendido/apagado o estrategias de control proporcional [3]. Los controladores de encendido y apagado (control ON-OFF) conducen a una pérdida significativa durante el período de desconexión debido a que se debe reiniciar el proceso y existe una migración de refrigerante durante este ciclo ${ }^{[4]}$.

Labus [5] llevó a cabo un control de supervisión para enfriadoras de absorción de pequeña potencia, en las cuales utilizó dos estrategias de control óptimas: la primera se encuentra basada en la modelación avanzada con Redes Neuronales Artificiales (RNA), mientras que la segunda utiliza métodos de optimización con Algoritmos Genéticos (AG), además, demuestra que estas dos estrategias pueden ser implementadas para el control online de sistemas de aire acondicionado con enfriadoras de absorción.

El esquema de control conocido como de temperatura de generación constante, se emplea, en configuraciones de climatización solar con caldera como sistema auxiliar, esta disposición, permite apoyar energéticamente el lazo de agua caliente, normalmente contra el depósito de alta temperatura. Esta configuración ha sido analizada en profundidad por Kohlenbach ${ }^{[6]}$ con un equipo de absorción de simple efecto de Agua y Bromuro de Litio de 10 kW, de la firma Phönix SonnenWärme AG [7]. El sistema analizado por Kohlenbach ${ }^{[6]}$ permite el acoplamiento directo entre el campo de captadores y el equipo de absorción, al hacer un baipás al depósito. Esta es una estrategia que permite los arranques tempranos por la mañana. Las diferentes estrategias de control (PID y ON-OFF) han sido ampliamente discutidas en la literatura por Kohlenbach [6]; el cual concluye, tras un análisis por simulación, que la mejora en el consumo eléctrico de diferentes estrategias de control de bombas en los circuitos de agua caliente (ON-OFF y PID) redunda 
en un $6 \%$, al mismo tiempo, que indica como infructuosa la búsqueda de la misma conclusión mediante la experimentación, al no encontrar en ese caso diferencias significativas en su campaña de ensayos. Con respecto al agua helada, Kohlenbach ${ }^{[6]}$, aplica técnicas clásicas de control ON-OFF, P, PI y PID, con los cuales ajusta la abertura de una válvula de tres vías que se encuentra a la entrada del generador de la Máquina de Absorción y que varía el flujo de agua caliente para variar la temperatura del agua helada. Kohlenbach ${ }^{[6]}$ además de los controles realizados al flujo de los circuitos de agua helada y agua caliente, controla la velocidad del ventilador de la torre de enfriamiento con un control PID.

Las reglas de lógica difusa para un controlador PID en un sistema de control para un refrigerador de absorción, fueron introducidos por Ogawa et al. ${ }^{[8]}$. El sistema controlado fue adaptado para someter a la temperatura de salida del medio de enfriamiento por medio de un control por lógica difusa, en el cual se ajusta la cantidad de calor suministrado al generador.

En Chile desarrollaron la Optimización de un Sistema de Refrigeración por Absorción asistido por Energía Solar al utilizar TRNSYS ${ }^{\circledR}$. El caso de estudio considerado en esta simulación, correspondió a un sistema de refrigeración solar por absorción que atiende una demanda de aire acondicionado sujeta al clima de la ciudad de Santiago. A partir de la simulación realizada en el entorno TrnBuild ${ }^{\circledR}$ de TRNSYS ${ }^{\circledR}$ para determinar la demanda de aire acondicionado de la vivienda en estudio, se obtuvieron resultados que indican que esta demanda se inicia a partir de agosto y culmina en el mes de abril, con períodos críticos para los meses de diciembre y enero, en los cuales, se produce la carga máxima de 23 kW (6.55 TR). Un controlador ON-OFF que actúa sobre la bomba del sistema para controlar el encendido y apagado del circuito de agua caliente. Posee un corte por alta temperatura, el cual se activa si la temperatura de entrada al colector es mayor a $98^{\circ} \mathrm{C}$. Se han considerado $2{ }^{\circ} \mathrm{C}$ y $0.15{ }^{\circ} \mathrm{C}$ como valores de upper dead-band y lower dead-band, respectivamente ${ }^{[9]}$.

Lygouras et al. [10], realizaron la implementación de un controlador por lógica difusa de estructura variable para un sistema de aire acondicionado con energía solar. Dos motores de corriente continua se utilizan para manejar la bomba del generador (agua caliente) y la bomba de alimentación (agua helada) del sistema de aire acondicionado con energía solar. Dos estrategias 
de control diferentes fueron aplicadas para controlar la regulación de la velocidad de rotación de los motores de corriente continua: el primero es un controlador difuso puro, su salida es la señal de control para el controlador del motor de corriente continua. Una matriz fuzzy 7 x 7 asigna la salida del regulador con respecto al valor del error y la derivada del error. El segundo es un controlador de dos niveles. El nivel inferior es un controlador PID convencional, y el nivel más alto es un controlador difuso que actúa sobre los parámetros del controlador de nivel bajo. La contribución de este diseño es, que en el sistema de control, la lógica difusa se implementa mediante software y un microcontrolador de 16-bits de bajo costo.

En Estados Unidos se proponen dos estrategias de control para un sistema de aire acondicionado con energía solar, con estas estrategias es posible generar un funcionamiento óptimo y una disminución en los costos operativos. El problema se resuelve al utilizar procedimientos de algoritmos genéticos implementados en MATLAB ${ }^{\circledR}$, para identificar las características de pay-off entre el costo diario de la energía, la carga térmica de la zona y la calidad del aire interior ${ }^{[11]}$. Utilizar algoritmos genéticos es más eficiente cuando el problema de optimización no es suave y uniforme o cuando la función de coste es ruidosa. La interacción entre las simulaciones del modelo y la herramienta de optimización (procedimiento de algoritmos genéticos en $\left.M A T L A B{ }^{\circledR}\right)$ se continúa hasta encontrar el resultado óptimo. Se debe tener en cuenta que cada modelo de componentes del sistema HVAC ha seguido un modelo conocido y validado en la literatura ${ }^{[12]}$.

\section{Disposición física del sistema}

Aunque en Colombia los sistemas de absorción no son nuevos, la mayoría emplean quemadores a gas para los sistemas de calentamiento de agua lo que ha generado un vacío en el uso de energías renovables como colectores solares o quemadores de biomasa. Este proyecto busca analizar un sistema de refrigeración por absorción, de efecto simple y de $\mathrm{LiBr}-\mathrm{H}_{2} \mathrm{O}$, al utilizar colectores solares como fuente de calor, con el fin de analizar costos, reducciones en la emisión de gases de efecto invernadero, entre otros. La Figura 1 presenta la disposición física del equipo a implementarse dentro del bloque 24 de la Universidad Pontificia Bolivariana. 
La máquina de absorción, marca Lucy Solar, a instalar dentro del sistema de refrigeración posee una potencia térmica de $11.5 \mathrm{~kW}$; adicionalmente, el sistema está dividido en tres zonas principales: zona de agua caliente, zona de agua helada y zona de agua fría. La zona de agua caliente cuenta con 12 colectores solares de marca Lucy Solar tipo Heat pipe ubicados en un arreglo serie-paralelo en grupo de 2 colectores, con el fin de garantizar un correcto equilibrio hidráulico y así prevenir una pérdida de caudal significativa, ya que la bomba que impulsa el agua por la tubería necesitaría una mayor cabeza para pasar el agua por cada uno de los tubos si se conectaran en serie todos los colectores; mientras que en la conexión serie paralelo el agua debe pasar por 2 colectores simultáneamente para calentarse, que permite seleccionar una bomba de menor potencia que a su vez cumpla con los valores establecidos de caudal, donde se recomiendan valores entre 0.1 y $0.25 \mathrm{l} / \mathrm{min} / \mathrm{m}$.

Dichos colectores serán los encargados de calentar el agua a la temperatura de activación de la máquina de absorción $\left(85^{\circ} \mathrm{C}\right)$, almacenándola en un tanque aislado de $1000 \mathrm{l}$, a través de la recirculación por medio de una motobomba de funcionamiento ON-OFF; por esta razón el arreglo debe contar con válvula ruptoras de vacío, válvula de venteo, válvula de seguridad y sensores de temperatura a la entrada y la salida de los colectores con el fin de garantizar la integridad de los mismos ante las altas temperaturas del agua y la generación de vapor que aumente drásticamente la presión interna de la tubería. Adicionalmente, para garantizar que la temperatura de entrada del chiller se encuentre en el rango adecuado, se empleará una caldera de paso alimentado por gas natural que cumplirá la función de apoyo, especialmente en horas de la mañana, en los momentos que se requiera un arranque rápido del sistema.

Para la zona de agua fría se empleará una torre de enfriamiento de 15 TR, marca Glaciar, con su respectiva motobomba, debido a que los sistemas de absorción por $\mathrm{LiBr}-\mathrm{H}_{2} \mathrm{O}$, pueden sufrir cristalización ante determinadas condiciones de temperatura, lo que obliga, a detener el equipo hasta que logre de nuevo su fusión, por lo cual se hace necesario emplear sistemas de rechazo de calor que permitan mantener las temperaturas en zonas no críticas. 


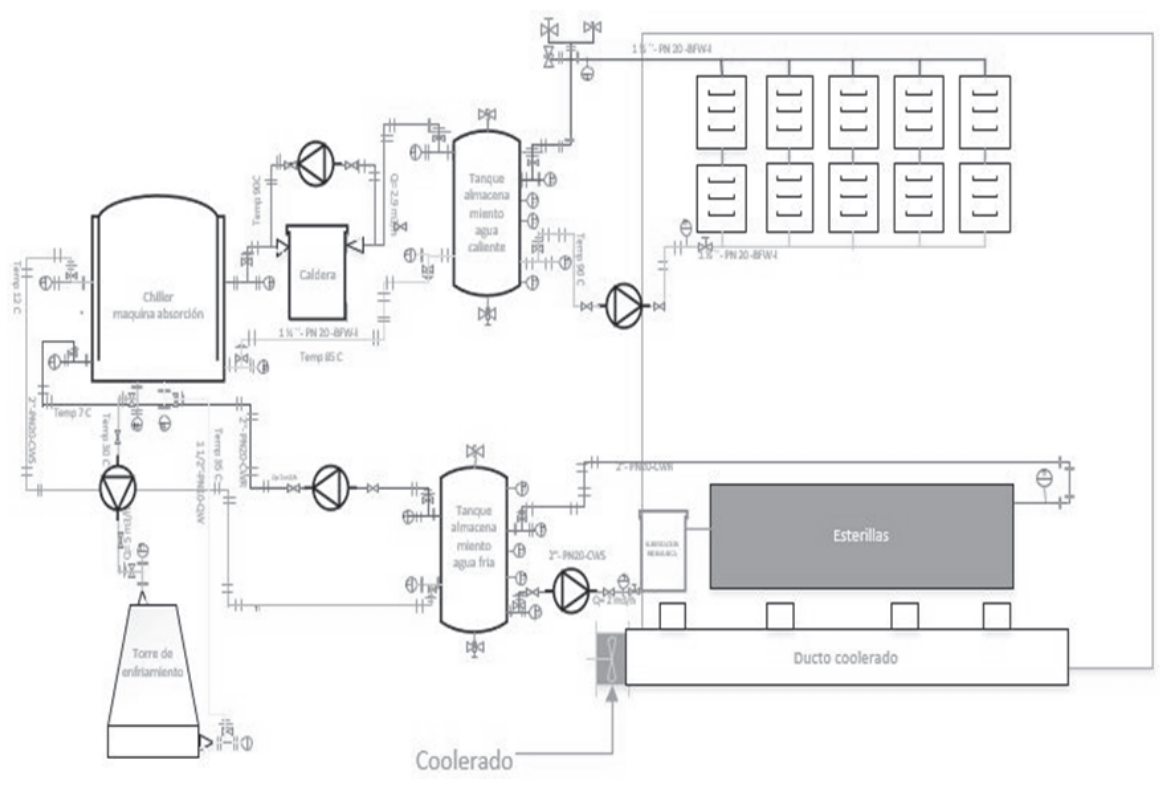

FIGURA 1. DISPOSICIÓN FÍSICA EQUIPOS AIRE ACONDICIONADO SOLAR

Por último, la zona de agua helada se encuentra conformada por un tanque aislado de $1000 \mathrm{I}$, para almacenar el agua de salida del chiller de absorción, una bomba de salida y un sistema de enfriamiento en recinto. Para este fin y con el propósito de incrementar la temperatura de evaporación en el ciclo de refrigeración por absorción, se ha optado por la integración de tecnologías energéticamente eficientes de pared radiante, usando esterillas de agua helada. Los sistemas de refrigeración de los acondicionadores por aire, operan con temperaturas bajas. Una relación típica de temperaturas entre el local y la temperatura del evaporador para un sistema sólo-aire es de $7^{\circ} \mathrm{C}$ para el aire y $5^{\circ} \mathrm{C}$ para el evaporador. En un sistema de pared radiante esta relación es de $15^{\circ} \mathrm{C}$ para el agua helada y $13^{\circ} \mathrm{C}$ para el evaporador.

Dado que los sistemas de pared radiante absorben básicamente las cargas térmicas de calor sensible, es necesario instalar un sistema de ventilación que permita evacuar la carga latente o de humedad del recinto, además de los elementos contaminantes que deban ser eliminados. Sin embargo, como se ha mencionado anteriormente este requiere un caudal mucho menor de aire. 
Por lo tanto, se ha seleccionado una tecnología energéticamente eficiente para el sistema de ventilación. Este está compuesto por un enfriador evaporativo indirecto M50 de marca COOLERADO, que reduce la temperatura del aire exterior a través del fenómeno de enfriamiento evaporativo, pero sin el consecuente aumento de la humedad de la corriente de aire que ingresa al recinto. Esto se logra gracias a una tecnología que usa un intercambiador de calor entre la corriente de aire que se humedece y enfría, y otra corriente de aire que es enfriada por esta, pero no recibe humedad.

La climatización por enfriamiento evaporativo o adiabático es un proceso que enfría el aire cuando se evapora el agua; el calor latente de evaporación se absorbe del cuerpo del agua y del aire de los alrededores; como resultado, tanto al agua como al aire se enfrían durante este proceso.

Para el cálculo de los diámetros de las tuberías de cada uno de los tramos del sistema:

1. Colectores- tanque recolección agua caliente

2. Tanque almacenamiento agua caliente-chiller absorción (incluido calentador)

3. Chiller absorción-tanque almacenamiento agua fría

4. Tanque almacenamiento agua fría-sistema esterillas

5. Chiller absorción-torre refrigeración

Se empleó la ecuación de Reynolds,

$$
\frac{P_{1}}{\gamma}+\alpha_{1} \frac{V_{1}^{2}}{2 g}+Z_{1}+H_{B}=\frac{P_{2}}{\gamma}+\alpha_{2} \frac{V_{2}^{2}}{2 g}+Z_{2}+h_{f},
$$

que asociada a la ecuación de Swanne Jain para el cálculo de pérdidas, y al evaluar en el software EES, dan como resultado:

$$
\begin{aligned}
& \operatorname{Din}_{\text {tramo_}_{1}}=0.02267 \mathrm{~m} \\
& \operatorname{Din}_{\text {tramo_ }_{2}}=0.01839 \mathrm{~m} \\
& \operatorname{Din}_{\text {tramo_3 }_{3}}=0.01973 \mathrm{~m} \\
& \operatorname{Din}_{\text {tramo }_{5}}=0.02166 \mathrm{~m}
\end{aligned}
$$

Para el tramo 4, aún se requiere conocer los diámetros de las esterillas para pared radiante a instalar ya que este es un valor relevante en el cálculo de los diámetros y los tamaños de la tubería 


\section{Resultados y Análisis}

Luego de analizar las cargas internas del recinto en diferentes horas laborales y desarrollar un modelo en SketchUp Pro ${ }^{\circledR}$ y empleando OpenStudio ${ }^{\circledR}$ y EnergyPlus ${ }^{\circledR}$, se logró determinar el comportamiento de la carga térmica con respecto a datos meteorológicos reales de la ciudad de Medellín en cada uno de los espacios del bloque a evaluar. La Figura 2 ilustra la carga térmica del recinto a partir de las cargas internas del recinto (Figura 3 ) y las cargas debidas a radiación solar, como se puede observar para la primera zona los equipos electrónicos correspondientes a equipos de cómputo y televisores ocupan la mayor parte de las cargas internas en el recinto.

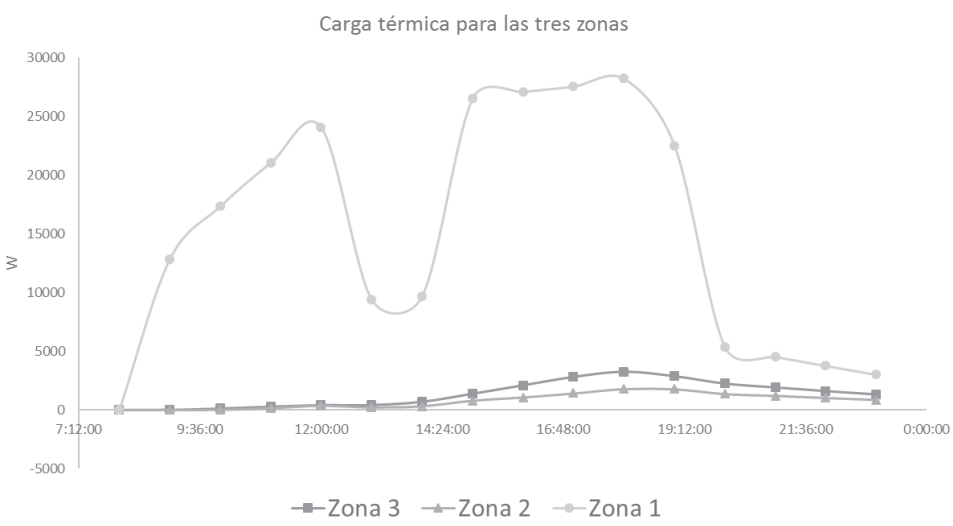

Figura 2. CARGA térMica PARA LAS TRES ZONAS DEL BLOQUE 24

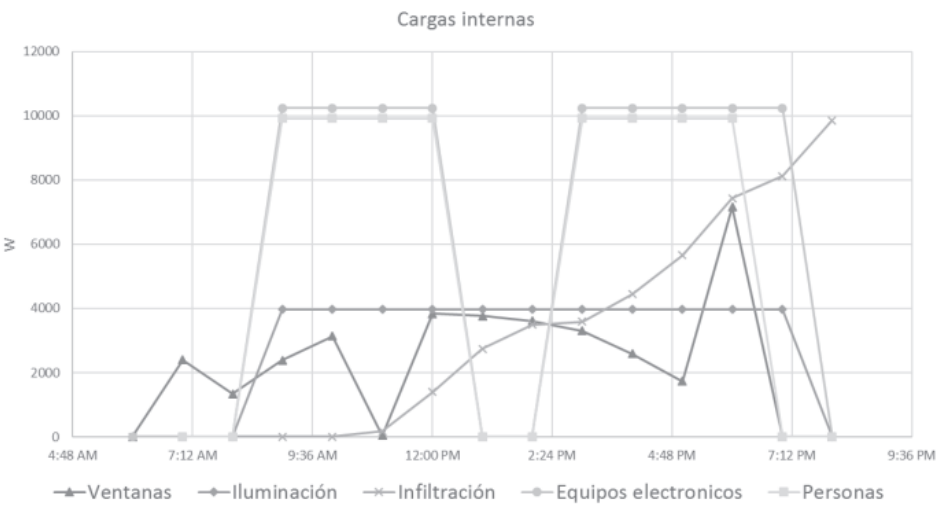

FIGURA 3. CARGAS INTERNAS DEL RECINTO 
Como se puede observar, la zona que presenta mayor carga térmica es la zona 1, dicha zona contiene la mayor cantidad de cargas internas y la cual se quiere climatizar. Se puede observar además que durante las horas de descanso (12.00 pm - 2:00 pm) dicha carga térmica disminuye en cierta proporción, pero es debido a que durante esa hora cierto personal sale del bloque y por tanto disminuye la carga interna producida por las personas. Durante las horas de la tarde, la carga interna aumenta y la transmisión de calor debe aumentar, debido a que, en horas de la tarde, mientras el sol se oculta, la carga por radiación aumenta. Para algunos días del año, la carga por infiltración permanecerá hasta ciertas horas de la noche, esta carga varía y es incierta, ya que se ve influenciada por las condiciones climáticas del lugar como la velocidad y dirección del viento, además del diferencial de temperaturas y presión que pueda existir entre el interior del bloque y el exterior.

Para realizar la simulación del sistema de control, se integraron los resultados de la evaluación de la carga térmica con el ciclo de absorción de simple efecto con $\mathrm{LiBr} /$ Agua y los subsistemas de colectores solares, torre de enfriamiento, distribución de agua helada, acumulación de agua helada y caliente y en la enfriadora auxiliar; al utilizar el programa computacional TRNSYS ${ }^{\circ}$. La modelización se llevó a cabo mediante la parametrización de los componentes que ofrece el programa en la librería TESS.

Para los datos meteorológicos se procesaron datos reales de la ciudad de Medellín (en donde existen varias estaciones meteorológicas) a través del software Meteonorm ${ }^{\circledR}$, obtenidos por medio del Laboratorio CALAIRE de la Universidad Nacional de Colombia, cerca de la UPB, para que el software TRNSYS ${ }^{\circledR}$ los procesara en los formatos requeridos.

La simulación del sistema de control de la instalación demostrativa, llevada a cabo en el software TRNSYS ${ }^{\circledR}$, muestra la aplicación de un control PI para la variación de la velocidad del ventilador de la torre de enfriamiento, se observan 2 controladores PI Difusos, uno realizado en la parte solar, para la variación del flujo de agua helada por medio de una válvula de tres vías que está conectada a la unidad manejadora (Fan-coil) de agua helada y otro para la variación de flujo de agua helada, a través de una motobomba; y muestra dos controladores ON-OFF, uno para la motobomba de agua caliente de los colectores solares, la cual es desconectada en la noche para que no sean subutilizados y otro para el calentador auxiliar, que siempre está encendido 
para alcanzar una temperatura de $90{ }^{\circ} \mathrm{C}$ en el generador del chiller de absorción, a menos que los colectores solares logren llegar a esta temperatura en el día, en este caso, el Calentador Auxiliar se apaga inmediatamente. La Figura 4 muestra la disposición y los elementos de control dependientes de cada uno de los equipos a instalar dentro del sistema de aire acondicionado solar diseñados dentro del software TRYNSYS ${ }^{\circledR}$.

En la Figura 5, se puede observar el comportamiento de la temperatura al interior del recinto, a partir de 2 controles PI Difusos realizados en el software $M A T L A B{ }^{\circledR}$, implementados en 2 válvulas, una instalada a la salida del tanque de agua fría, y la otra a la salida de la enfriadora auxiliar. Estas válvulas regulan el flujo de agua helada que entra al Fan-coil, el cual es el actuador final que, por medio de un ventilador, expulsa aire frío, el cual es generado gracias al agua helada producida por el chiller de absorción y la enfriadora auxiliar.

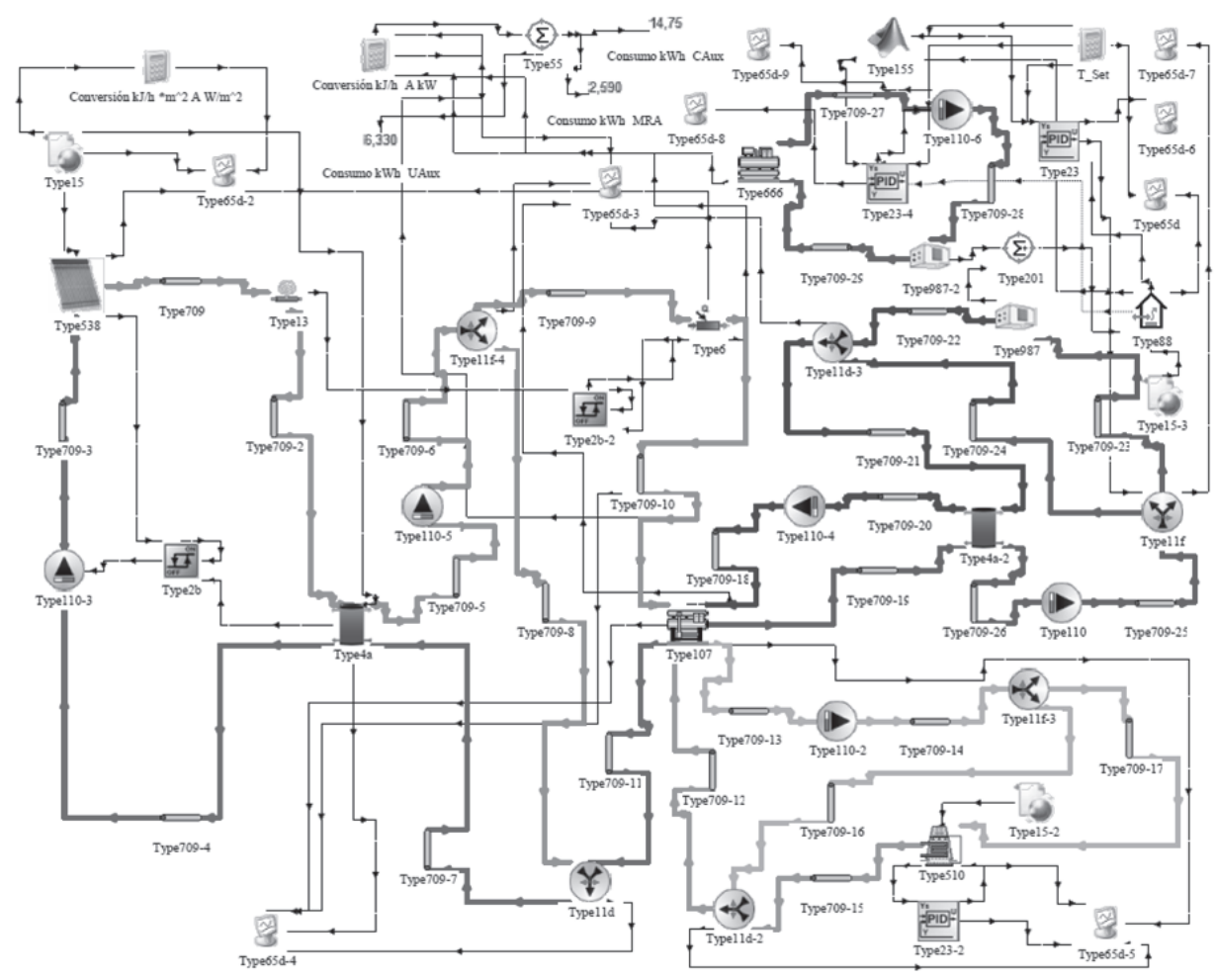

FIGURA 4. SIMULACIÓN DEL SISTEMA DE AIRE ACONDICIONADO SOLAR Y CICLO DE REFRIGERACIÓN POR ABSORCIÓN 


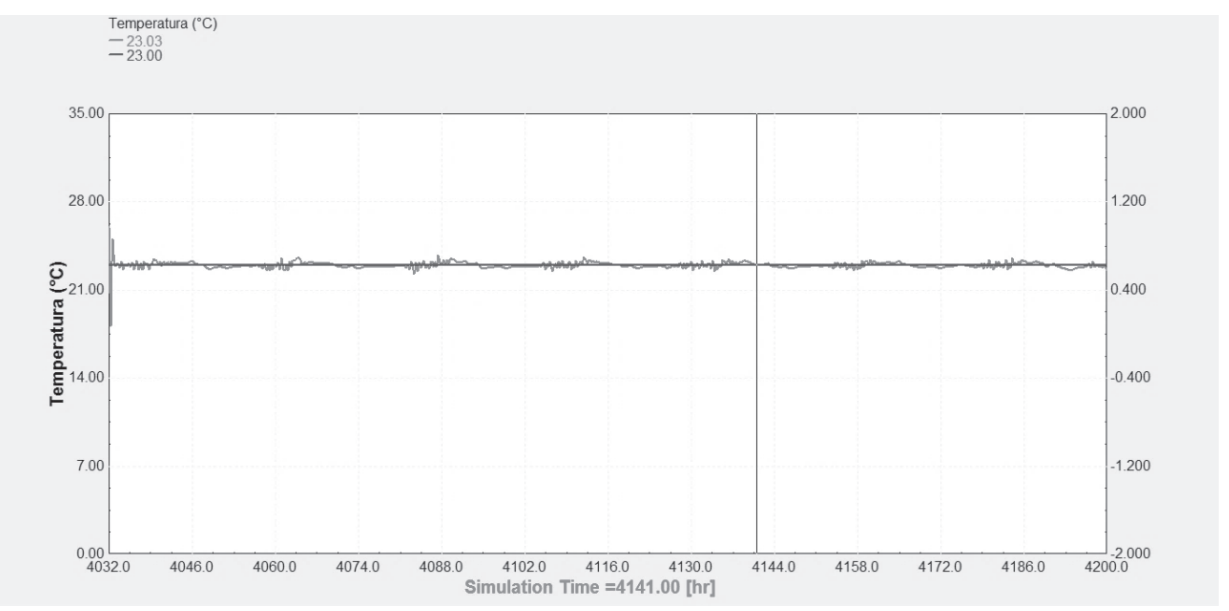

FIGURA 5. TEMPERATURA INTERNA DEL SISTEMA ANTE CONTROL PI DIFUSO

\section{Conclusiones}

El Sistema de Refrigeración Solar, se encuentra en implementación dentro del bloque 24 de la Universidad Pontificia Bolivariana, lo que permitirá generar un banco de pruebas para diferentes algoritmos de control, mezclas de gases refrigerantes y que a su vez permita analizar las ventajas de este tipo de sistemas en comparación con los sistemas de aire acondicionado tradicional

A partir de los resultados obtenidos durante la fase de experimentación y simulación, se puede determinar el alto impacto que este tipo de sistemas pueden tener, ya que en lugares, donde no sólo se requiere acondicionamiento de aire, sino que a su vez se requieran sistemas de cogeneración o trigeneración, el sistema puede ser fácilmente escalable y el calor rechazado puede ser reutilizado en procesos donde se requiera calentamiento de agua u otros procesos.

El sistema de aire acondicionado solar simulado presenta un comportamiento no lineal en la parte de acondicionamiento de aire, ya que las condiciones ambientales y las cargas térmicas influyen, perturban y cambian constantemente en el tiempo, es por esto que una técnica de control inteligente (PI Difuso) es bastante determinante, ya que genera un menor consumo de energía eléctrica por la acción de control suave aplicada sobre los actuadores 
y, además, logra estabilizar en menos tiempo la variable temperatura, la cual es la variable controlada.

Las simulaciones, al emplear un software como TRNSYS ${ }^{\circledR}$, EnergyPlus ${ }^{\circledR}$ y OpenStudio ${ }^{\circledR}$; pueden permitir aproximaciones bastante cercanas a la realidad en sistemas de refrigeración por absorción lo que las convierte en herramientas poderosas en etapas de diseño

\section{Referencias}

[1] A.R.TrottyT.Welch, Refrigerationandair-conditioning,3rded.Oxford; Boston, Mass.: Butterworth Heinemann, 2000, previous ed.:1989.

[2] J. Meza, J. Gonz_alez, y A. Khan, Experimental assessment of a solar assisted air conditioning system for applications in puerto rico," ASME Proceedings of the Solar Energy Division 8, vol. 1, pp. 149\{154, 1998.

[3] J. Fernández-Seara y M. Vázquez, Study and control of the optimal generation temperature in nh3-h2o absorption refrigeration systems," Applied Thermal Engineering, vol. 21, no. 3, pp. $343\{357,2001$.

[4] J. Wang y Y. Wu, Start-up and shut-down operation in a reciprocating compressor refrigeration system with capillary tubes," International Journal of Refrigeration, vol. 13, no. 3, pp. 187-190, 1990.

[5] J. Labus, Modelling of small capacity absorption chillers driven by solar thermal energy or waste heat," Ph.D. dissertation, Universitat Rovira I Virgili, Tarragona, Septiembre 2011.

[6] P. Kohlenbach, Solar cooling with absorption chillers: Control strategies and transient chiller performance,"Ph.D. dissertation, Technischen Universität Berlin, Enero 2006.

[7] PhönixSonnenWärmeAG, |Phönix sonnenwärme ag," 2015. [En línea]. Disponible en: http://www.sonnenwaermeag.de/.

[8] A. Ogawa, K. Hitomi, M. Maekawa, K. Yoshii, H. Arima, y E. Enomoto, Control system for absorption refrigerator," Japan Patent US5 138846 A, Aug 18, 1992, uS Patent $5,138,846$.

[9] H. Vidal y D. P. Mansilla, Optimización de un sistema de refrigeración por absorción asistido por energía solar utilizando trnsys,"Revista Frío y Calor”, vol. 1, no. 99, pp. 5-11, Noviembre 2009. 
[10] J. Lygouras, P. Botsaris, J. Vourvoulakis, y V. Kodogiannis, IFuzzy logic controller implementation for a solar air-conditioning system," Applied Energy, vol. 84, no. 12, pp. 1305-1318, 2007.

[11] J. A. Wright, H. A. Loosemore, y R. Farmani, Optimization of building thermal design and control by multi-criterion genetic algorithm," Energy and Buildings, vol. 34, no. 9, pp. 959-972, 2002, a View of Energy and Building Performance Simulation at the start of the third millennium.

[12] J. House y T. U. o. I. Smith, A system approach to optimal control for hvac and building systems, "in Conference: Annual meeting of the American Society of Heating, Refrigeration and Air-Conditioning Engineers, Inc. (ASHRAE), San Diego, CA (United States), 24-28 Jun 1995; Other Information: PBD: 1995; Related Information: Is Part Of ASHRAE transactions 1995: Technical and symposium papers. Volume 101, Part 2; PB: 1497 p., vol. 101. American Society of Heating, Refrigerating and Air-Conditioning Engineers, Inc., Atlanta, GA (United States), Diciembre 1995, p. 1497. 